\title{
Tabularia
}

TABULARIA Sources écrites des mondes normands médiévaux

\section{Comment lisait-on une lettre au Moyen Âge ? Le témoignage du Roman du Mont Saint-Michel}

How to read out a letter in the Middle Ages? The testimony of the Roman du Mont Saint-Michel

\section{Catherine Bougy}

\section{CpenEdition}

Journals

Édition électronique

URL : http://journals.openedition.org/tabularia/2470

DOI : $10.4000 /$ tabularia. 2470

ISSN : 1630-7364

Éditeur :

CRAHAM - Centre Michel de Boüard, Presses universitaires de Caen

Référence électronique

Catherine Bougy, "Comment lisait-on une lettre au Moyen Âge ? Le témoignage du Roman du Mont Saint-Michel », Tabularia [En ligne], Sources en ligne, mis en ligne le 02 juillet 2003, consulté le 01 mai 2019. URL : http://journals.openedition.org/tabularia/2470 ; DOI : 10.4000/tabularia.2470 


\title{
Comment lisait-on une lettre au Moyen Âge? Le témoignage du Roman du Mont Saint-Michel
}

\section{How to read out a letter in the Middle Ages? The testimony of the Roman du Mont Saint-Michel}

\author{
Catherine BOUGY \\ CRISCO/OUEN \\ Université de Caen Basse-Normandie \\ bougy@mrsh.unicaen.fr
}

\section{Résumé:}

Une digression du Roman du Mont Saint-Michel, texte en vers français de Guillaume de Saint-Pair, moine de l'abbaye, constitue un témoignage précis de la façon dont, au XII siècle, il était fait lecture d'une lettre à un haut personnage. Le document officiel en latin, reconnaissable comme tel par sa formule finale, valei, est une lettre du pape qui se présente probablement sous la forme d'un rouleau de parchemin. Il est confié à un chapelain qui le développe, l'examine attentivement et récite à Richard I ${ }^{\text {er }}$ de Normandie, ce qu'il entend $\mathrm{du}$ texte, lui en traduisant sans faillir le contenu.

Mots-clés : Charte, lettre, bref, privilège, rouleau, chapelain, récitation, traduction, formules.

\begin{abstract}
:
A digression in the Roman du Mont Saint-Michel, a French poem by William of Saint-Pair, monk at Mont Saint-Michel, is a particularly good example of how in the twelfth century one should read out a letter to a high ranking person. The official document in Latin, recognisable as such by its valediction, valei, is a papal letter presented probably in the form of a parchment roll. It is entrusted to a "chaplain" who "unrolls» it, examines it attentively and "recites" to Richard I of Normandy what he « understands » of the text, while translating it for him without «losing» the contents.
\end{abstract}

Keywords: Charter, letter, writ, privilege, roll, chaplain, recitation, translation, formulas. 
Pour l'élaboration de la version française des textes fondateurs du Mont SaintMichel, le moine Guillaume de Saint-Pair ${ }^{1}$, qui écrivait vers 1155 , sous l'abbatiat de Robert de Torigni ${ }^{2}$, disposait de la copie qui en avait été récemment faite sur ordre de l'abbé Geoffrey (1149-1150)³ dans le Cartulaire du Mont Saint-Michel ${ }^{4}$.

Il s'agit, pour le livre I ${ }^{\mathrm{er}} \mathrm{du}$ Roman du Mont Saint-Michel, de la Revelatio ecclesioe sancti Michoelis in Monte qui dicitur Tumba (Cartulaire, Avranches, Bibl. mun., ms 210 fol. $\left.5 r^{\circ}-10 r^{\circ}\right)$, qui relate les apparitions de l'archange saint Michel à l'évêque d'Avranches, Aubert, pour lui ordonner d'édifier sur le Mont Tombe un sanctuaire à lui dédié; l'événement est daté de 709, le texte a été écrit au début du IX ${ }^{\mathrm{e}}$ siècle. Le livre II a pour source l'Introductio monachorum et miracula insigniora per beatum Michoelem archangelum patrata in ecclesia que dicitur Tumba, in periculo maris sita, nomine ipsius Archangeli fabricata, texte du XI ${ }^{\mathrm{e}}$ siècle, qui expose les liens existant entre le sanctuaire et les « ducs de Normandie, de Rollon à Guillaume le Bâtard, en accordant une large place au remplacement, en 966, sur ordre de Richard ${ }^{\text {er }}$ de Normandie, des chanoines qui assuraient le culte du saint par des moines bénédictins ( $\mathrm{ms} 210$ fol. $10 \mathrm{r}^{\circ}-19 \mathrm{r}^{\circ}$ ); il insiste également sur les donations faites au Mont par les «ducs », transposant avec une extrême fidélité deux actes, l'un de Richard II, (daté entre 1022 et 1026), le second de Robert le Magnifique (daté de 1027-1037) ${ }^{5}$. Le livre III a pour source certains des récits de miracles qui font suite à l'Introductio monachorum ${ }^{6}$.

Une confrontation des 4106 octosyllabes qui nous sont parvenus et des textes latins rédigés à différentes époques, qui présentent aux lecteurs instruits l'histoire du sanctuaire, montre que le plus souvent la version française est d'une parfaite fidélité à l'original latin, jusqu'à en être parfois une transposition terme à terme.

Mais, penchant naturel ou désir, légitime chez un auteur, de se ménager des plages de création personnelle, le Roman du Mont Saint-Michel offre aussi un certain nombre de digressions ${ }^{7}$, dont certaines s'étendent sur plusieurs centaines de vers, tenant parfois du catalogue... Le lecteur moderne ne s'en plaindra pourtant pas, qui y trouve des informations sur des sujets aussi variés que les poissons de la

1. Saint-Pair-sur-Mer: dép. Manche, cant. Granville.

2. 1154-1186.

3. Mai 1149-décembre 1150. Sur la question de la datation du Cartulaire, cf. KEATS-ROHAN, Katharine S. B. «Bibliothèque municipale d'Avranches, 210: Cartulary of Mont-Saint-Michel », Anglo-Norman Studies XXI (1999), p. 95-112; et id., «Une charte de l'abbé Mayeul de Cluny et la réforme du Mont Saint-Michel» in La Normandie vers l'an mil, François de BEAUREPAIRE et Jean-Pierre CHALINE (éd.), Rouen, Société de l'Histoire de Normandie, 2000, p. 159-169.

4. Avranches, Bibl. mun., ms 210.

5. Publiés dans le Recueil des actes des ducs de Normandie de 911 à 1066, de Marie Fauroux, Caen, Société d'Impression Caron, 1961, sous les $n^{\text {os }} 49$ et 73.

6. Cf. Les Curieuses recherches du Mont Saint-Michel par Dom Thomas Le Roy, éd. Eugène de RoBILLARD de BeAurepaire, Mémoires de la Société des Antiquaires de Normandie, Caen, Le Gost-Clérisse, 1878, t. I, p. 407-419 (Revelatio), p. 419-435 (Introductio monachorum) et p. 435-464 (miracula).

7. Certaines sont présentées comme telles par l'auteur. Cf. Roman du Mont Saint-Michel, livre I, v. $417-$ 419: Or feron ci digression Quer un petit conter volum Quel fut li monz primes et pois: «Nous allons maintenant faire une digression car nous voulons décrire un peu le Mont à ses origines et par la suite». 
baie du Mont Saint-Michel, la consécration d'une église, les vêtements portés par un évêque sur son lit de $\operatorname{mort}^{8}$...

Aux vers 1787-1854 du livre II du Roman, l'une de ces digressions présente la scène détaillée de la lecture d'une lettre. Les circonstances en sont les suivantes: Richard I ${ }^{\text {er }}$ de Normandie ${ }^{9}$, petit-fils de Rollon est mécontent de la tiédeur religieuse et des mœurs dissolues des chanoines chargés du culte de l'archange sur le Mont. Après consultation de l'archevêque de Rouen ${ }^{10}$ et du roi de France ${ }^{11}$, il a envoyé des messagers au pape Jean XIII ${ }^{12}$ pour l'informer de cette situation et le consulter sur son projet de les remplacer par des moines bénédictins ${ }^{13}$. Le pape et ses cardinaux lui ont donné leur accord; les envoyés du duc rapportent à leur seigneur la réponse du souverain pontife, consignée dans une lettre.

Nous donnons de ces vers la version qui figure dans le manuscrit Additional 10289 de la British Library (appelé ici $A$ ), fol. $30 v^{\circ}-31 v^{\circ}$, du XIII siècle, avec en notes celles des variantes du manuscrit Additional $26876(B)$, fol. $47 \mathrm{r}^{\circ}-48 \mathrm{r}^{\circ}$, du XIVe siècle, qui présentent de l'intérêt pour la compréhension du texte. C'est sur $A$, la première des deux versions existantes du Roman, plus ancienne et plus fiable que $B$, que nous nous fondons pour l'établissement du texte ${ }^{14}$.

Entretant a molt vivement

Li quens fait son porchacement:

De l'archevesque Huun out

1790 Primes un brief, tel cum il vout;

Del rei Lohier un tel en ra

Come il meïsme demanda.

Quant ses bries a, homes a quis,

8. Roman du Mont Saint-Michel, livre I, v. 467-474, v. 749-1132, v. 1223-1234.

9. $942-996$.

10. Hugues, archevêque de Rouen, 942 (ou 943)-989.

11. Lothaire, $954-986$.

12. Jean XIII, 965-972.

13. L'auteur expose longuement les circonstances de l'installation de moines bénédictins au Mont SaintMichel, en insistant sur le processus de consultations et sur l'autorisation du pape, préalables à la décision de Richard $\mathrm{I}^{\mathrm{er}}$.

14. La seule édition française de l'œuvre de Guillaume de Saint-Pair, uniquement fondée sur le texte incomplet du manuscrit $A$ est celle de Francisque Michel Le Roman du Mont-Saint-Michel par Guillaume de Saint-Pair, in Mémoires de la Société des Antiquaires de Normandie $n^{\circ} 20,2^{e}$ série, $10^{\circ}$ volume de la collection, Paris, Derache/Didron; Caen, Hardel; Rouen, Le Brument, 1853. On signalera également une édition critique du texte de $A$, assortie de l'édition diplomatique du texte de $B$ par Paul REDLICH, Der Roman du Mont Saint-Michel von Guillaume de S. Paier, Wiedergabe der beiden Handschriften des Britischen Museum von Dr. Paul Redlich, in Ausgaben und Abhandlungen aus dem Gebiete der romanischen Philologie, XCII, Marburg, N.G. Elwert'sche Verlag Buchhandlung, 1894. La thèse non publiée de R. Graham BIRRELL, Le Roman du Mont-Saint-Michel by Guillaume de Saint-Pair, a critical edition presented for the degree of $P h$. D., Université d'Aberdeen, 1978, constitue la plus récente édition critique complète du texte (sans traduction); elle prend en compte le texte des deux manuscrits. 
Des plus sages de son païs:

1795 Son message lor encharga

Et a Rome les enveia.

Espleitié unt tant et esré

Que l'apostoile ourent trové.

Li apostoile de cel tens

1800 Out non Johan, si cum je pens.

Lor bries li livrent seielez:

Bruisiez les a et esguardez.

Par sei esteit enz en un brief

La vie as clers, de chief en chief.

1805 Enz en un altre aprof esteit

Ce que li dux li requiereit.

$A$ icels dous erent semblables

Li altre tuit et acordables ${ }^{15}$.

Quancque li quens li requiereit

1810 Par ses chartres que il diseit

Li apostoiles otria ${ }^{16}$

Et de sa part le conferma;

Joious en ert et liez forment.

Li cardinal tuit ensement

1815 Al duc runt bries tels enveiez

Cum par les suens li out preiez.

Quant li messages ont pris congié

Isnelement sunt repairié.

Chevaus ourent ad volenté:

1820 Par jornees ont tant esré

Qu'en Normendie sunt venu.

Li dus les vit, toz liez en fu;

De chief en chief li unt conté

Cum faitement ourent esré.

1825 Il apela un chapelain,

Le brief li mist enz en la main

Que cil aveient aporté;

Li clers l'a tost desvolepé.

Despleié l'a et esgardé,

1830 Puis l'a au comte recité:

Les saluz dist premierement

Trestot eissi cum les entent,

15. 1807-1808 absents dans $B$.

16. 1810 absent dans B; pour 1809-1812, B: Quanque li dus requis lia Laposteile li ostria Et de sa part le conferma. 
Puis a tot leit ${ }^{17}$ le brief avant,

Qu'il n'i falli ne tant ne quant.

1835 Li apostoiles li mandout

Par cez lestres que enveout ${ }^{18}$

Que del mont ost toz les chanoines

Et en leu d'els il mete moines,

Et les rentes aient al mont

1840 Que li chanoine tenu unt,

Et enz et fors li otreout ${ }^{19}$

Et de sa part le confermout ${ }^{20}$.

Et si aucuns le contredit

Il l'escumenge et maldit.

1845 De l'autre part, s'en l'abeie

Velt nul des clers muer sa vie

Bien li otreie de par Deu;

Iluec ${ }^{21}$ n'out plus quer n'en fut leu.

Fors le valei, a la parfin,

1850 Qui eirt escrit el parchemin.

A l'archevesque uns en ralout

Qui ce meïsmes commandout.

Li evesques d'Avrenches rout

Un brief qui cen reconfermout

«Pendant ce temps le comte s'activait à la réalisation de son projet: il avait d'abord obtenu de l'archevêque Hugues une lettre conforme à ses souhaits, puis une autre, de la part du roi Lothaire, telle qu'il l'avait demandée. Une fois en possession de ses lettres, il se mit en quête d'hommes, parmi les plus sages de son pays; il leur confia son message et les envoya à Rome. Ils firent tant et si bien qu'ils parvinrent auprès du pape. Le pape de l'époque portait, je pense, le nom de Jean. Ils lui remirent leurs lettres scellées : il en brisa les sceaux et les examina. Dans une lettre à part était relatée la vie des clercs dans ses moindres détails, dans une autre se trouvait ensuite la requête du comte au pape. Toutes les autres lettres étaient semblables à ces deux-là et concordaient avec elles.

Le pape, par des chartes qu'il dictait, donna son accord à toutes les demandes du comte et les confirma personnellement: il s'en réjouissait très vivement. Les cardinaux unanimes envoyèrent aussi au duc des lettres conformes aux demandes qu'il avait formulées dans les siennes.

17. A : Puis a tot leit; B: Puis a tot leu.

18. 1836 absent dans $B$.

19. A : Et enz et fors li otreout; B: Dehors et denz onques qu'il sont.

20. 1842 absent dans $B$.

21. A : Illueques nout plus; B: Illeuc nout plus. 
Après avoir pris congé, les messagers repartirent très vite: ils avaient des chevaux à volonté et, avançant par étapes, ils parvinrent enfin en Normandie. Le duc se réjouit fort de les voir ; ils lui firent en détail le récit de leur voyage.

Il appela un chapelain et remit entre ses mains la lettre qu'ils avaient apportée. Le clerc la déroula. Il la déploya et l'examina, puis en fit lecture au comte, en commençant par les salutations, exactement comme il les comprenait; puis il lui lut la lettre entièrement, sans omettre quoi que ce fût.

Le pape lui ordonnait, par cette lettre ${ }^{22}$ qu'il lui envoyait, de chasser du Mont tous les chanoines, de mettre à leur place des moines et de leur accorder les rentes attribuées au Mont, que les chanoines avaient occupé. Il lui donnait son accord sans restriction ${ }^{23}$ et le lui confirmait pour sa part. Et si quelqu'un contestait cette décision, il l'excommuniait et le maudissait. D'un autre côté, si l'un des clercs voulait rester dans l'abbaye en changeant sa façon de vivre, il l'y autorisait, au nom de Dieu. Il n'y avait rien de plus dans la lettre, car c'était inutile, sauf le vale tout à la fin, qui était écrit sur le parchemin.

Un autre parchemin contenant ces mêmes instructions était adressé à l'archevêque; l'évêque d'Avranches en reçut également un, qui confirmait aussi tout cela. »

Les circonstances et les conditions de la rédaction du message papal sont clairement exposées : en réponse à la demande du duc de Normandie, le pape a dit, c'està-dire qu'il a formulé à haute voix un avis (v. 1810). Noté par écrit sous la forme d'une charte ${ }^{24}$, celui-ci a été remis, avec les $b r e f s^{25}$ des cardinaux, aux messagers

22. Lettres, au pluriel, comme le latin litterce, désigne, du XI ${ }^{\mathrm{e}}$ au $\mathrm{XV}^{\mathrm{e}}$ siècle, « une épître, une missive, ce qu'on écrit à quelqu'un pour l'informer d'une nouvelle, etc. » (VON WARTBURG, Walther, puis ChAmbon, Jean-Pierre, puis CHAUVEAU, Jean-Paul, Französisches Etymologisches Wörterbuch, Bonn, Leipzig, Berlin, Bâle, 1928-... [ci-après: FEW], V, 377b, littera). Le Dictionnaire du Moyen Âge de Claude Gauvard, Alain de LIBERA, Michel ZINK (dir.), Paris, Quadrige/P.U.F., 2002., p. 829 , article «lettre», précise que «le terme de "lettre" ne désigne pas seulement la missive privée ou ex officio, mais s'applique également à des actes en forme, des "chartes", lesquelles ont ordinairement une rédaction épistolaire (avec adresse, salut, etc.).»

23. Enz et fors, littéralement «à l'intérieur et à l'extérieur» (des adverbes latins de même sens, intus et foras/foris) signifie donc "partout», «sans limites, sans restrictions, totalement». Cf., cités par Tobler, Adolf, LOMmatzsch, Erhard, Altfranzösisches Wörterbuch, Wiesbaden, Franz Steiner Verlag, 1925-1976, III, 738, ces vers d'un fabliau (Méon II, 3, 61) : «Bele fu [l'empereris] enz, bele fu fors, Bel ot lou cuer, bel ot lou cors: [l'impératrice] était belle à l'intérieur comme à l'extérieur [ "totalement»]. Son cœur était beau, son corps était beau».

24. Cf. pour le sens de ce terme BAUTIER, Robert-Henri, «Caractères spécifiques des chartes médiévales » in Chartes, sceaux et chancelleries. Études de diplomatique et de sigillographie médiévales, Paris, École des chartes, Mémoires et documents de l'École des chartes, 1990, 2 vol., cité par GAUVARD, DE LIBERA, ZINK, Dictionnaire du Moyen Âge, p. 267, article «charte» : «Pour l'homme du Moyen Âge, dire carta ou charta était à peu près aussi précis que de parler d'un papier». L'auteur de l'article (Laurent Morelle) insiste sur le caractère «flou » du terme, qui désigne un acte écrit, public ou privé, et peut aussi, de façon générale, s'appliquer à un privilège. Dans le passage, il peut s'agir du premier comme du second sens, dans la mesure où la lettre du pape s'accompagne d'un privilège accordé aux moines. Voir également, pour des éléments complémentaires sur les notions de charte et de lettre GUYOTJEANNIN, Olivier, PYCKE, Jacques, et TOCK, Benoît-Michel, Diplomatique médiévale, Turnhout, Brepols, 1993.

25. Cf. FEW I, 520ab, brevis, " court » : depuis le VIe siècle, le neutre breve a pris le sens de "court résumé, charte », d'où l'ancien français brief « lettre; registre à inscrire les droits, chronique ». Si letres est 
(v.1814-1817). Les envoyés du duc se hâtent de les rapporter à Richard (v. 1817-1821). Introduits auprès de leur maître, ils lui rendent compte de leur mission. Lecture lui est alors faite des documents (v. 1825-1854).

Richard I ${ }^{e r}$ ne lit pas lui-même le message, mais a recours à l'intermédiaire d'un chapelain (v. 1825). Faudrait-il en conclure qu'il ne savait pas lire, ou qu'il ne convenait pas à un homme de son rang de procéder lui-même à la lecture d'une lettre?

Sur le degré d'instruction du fils de Guillaume Longue Épée, on sait peu de choses, sinon, par Dudon de Saint-Quentin, que son père l'avait envoyé à Bayeux pour y apprendre la langue norroise, ce qui suggère qu'il était de langue romane. Mais Dudon ne précise pas s'il avait aussi appris à lire et à écrire auprès de son parrain, Bothon ${ }^{26}$.

Le détail du chapelain chargé de lire une lettre à un noble personnage se rencontre d'ailleurs dans d'autres textes ${ }^{27}$, ce qui indique qu'il s'agissait d'un procédé habituel, même lorsque le destinataire était instruit. Wace montre ainsi l'archevêque de Cantorbéry, Lanfranc, recevant une lettre de Guillaume le Bâtard que lui apporte le fils du roi, Guillaume le Roux, et la faisant lire: E Lanfran fist les letres liere ${ }^{28}$.

C'est que cet acte de lecture à haute voix, la récitation (cf. v. 1830 recité), faite par un tiers, permettait de procéder à la publication officielle d'un document important, ainsi que l'a précisé Gilduin Davy dans un article récemment paru dans Tabularia ${ }^{29}$.

Enfin, dans le cas précis de la scène décrite dans le Roman, on soulignera le valei (v. 1849) que le clerc n'omet pas de transmettre à son seigneur. Valei ${ }^{30}$, dans

un terme de sens très général tandis que brief désigne un court document officiel, dans le passage que nous étudions, brief et letres semblent synonymes.

26. Cf. DUdON DE SAINT-QUENTIN, De moribus et actis primorum Normannice ducum, éd. Jules LAIR, in Mémoires de la Société des antiquaires de Normandie, tome XXIII, Caen, Le Blanc-Hardel, 1865, IV, 68, p. 221-222.

27. Cf. Michel, Francisque, Relation du tournoi de Ham (XIII e siècle) p. 314, in Histoire des Ducs de Normandie et des rois d'Angleterre suivie de la relation du tournoi de Ham, SARRAZIN (éd.) Paris, 1840, (cité par TOBLER-LOMMATZSCH, II, 239-240, s.v. chapelain) : La demoisele de rechief Commence son message a dire Et dist : "Roïne faites lire Ceste lettre que je vous baille Que il convient que tost m'en aille. » La roïne prent en sa main La lettre et huce un capelain Qui li devise mot a mot. : «La noble jeune fille énonce à nouveau son message: "Reine, faites lire cette lettre que je vous apporte, car il faut que je m'en aille bientôt". La reine prend la lettre dans sa main et appelle un chapelain qui lui en expose le contenu mot pour mot».

28. Le Roman de Rou de Wace, éd. Anthony John Holden, Paris, A. et J. Picard, 1970, 1971, 1973, III 9349-9354 (vol. II, 1971, p. 233): Guilleme le Ros mer passa, coronez fu,. Xiii. anz regna. De par son pere seelé out a Lanfran un brief porté, l'archevesque de Cantorbiere, E Lanfran fist les letres liere. ("Guillaume le Roux traversa la mer; il fut couronné et régna treize ans. Il avait apporté à Lanfranc, l'archevêque de Cantorbéry, une lettre scellée par son père. Et Lanfranc fit lire la lettre»).

29. DAVY, Gilduin, «Le scribe, le droit et le prince : recherche autour de l'utilisation de l'écrit juridique par les ducs de Normandie de la fin du $\mathrm{X}^{\mathrm{e}}$ siècle à la fin du XI $\mathrm{X}^{\mathrm{e}}$ siècle», Tabularia: «La récitation du texte demeure un moment fondamental de la procédure de publication des actes juridiques la cour ducale [...]». [http://www.unicaen.fr/mrsh/crahm/revue/tabularia/davy.html]. Cf. également FEW, $\mathrm{X}, 150 \mathrm{a}$, recitare, fr. reciter " lire à haute voix »; " rapporter, faire connaître».

30. La graphie ei permet de différencier à l'écrit un é fermé d'un $e$ sourd. $B$ présente la leçon vale, où le $e$ final peut être interprété soit comme un é fermé (celui de clé) soit comme un e sourd (celui de l'article le). 
une lettre en latin, est la formule finale, littéralement «porte-toi bien ». Le fait que la charte du pape soit rédigée en latin fournit une explication supplémentaire à ce recours à un lecteur: on peut penser que, même instruit, le destinataire a besoin de connaître avec précision le contenu de la lettre; elle doit donc lui être traduite par un spécialiste qui lui en restituera toutes les subtilités et évitera les erreurs d'interprétation : c'est ici le rôle du chapelain, qu'il faut comprendre comme « secrétaire ${ }^{31}$; le terme est repris par clerc, qui cumule les sens de « homme d'église » et de «lettré» ${ }^{32}$.

Ce dernier va d'abord devoleper et desploier la lettre (v.1828-1829) ${ }^{33}$. Les deux verbes peuvent être considérés comme des synonymes, employés de manière redondante, pour décrire le geste du lecteur déroulant, afin d'en prendre connaissance ${ }^{34}$, un parchemin qui se présente sous la forme d'un rouleau : ainsi celui que, sur un des dessins encrés du Cartulaire, la duchesse Gonnor, épouse de Richard I ${ }^{\text {er }}$, tend à l'abbé du Mont à qui elle fait des donations de terres et de rentes pour son abbaye ${ }^{35}$.

D’autres textes médiévaux indiquent qu'une lettre pouvait aussi prendre la forme d'un quareignon, feuille de parchemin pliée en quatre. C'est un message ainsi présenté qu'un clerc scelle et place dans une boîte avant de l'apporter à son destinataire dans

31. L'emploi du déterminant indéfini devant le substantif indique qu'il ne s'agit pas ici du chapelain personnel du comte, chargé de dire la messe dans sa chapelle privée, de son chapelain, mais d'un des clercs chargés de son secrétariat. Cf. la définition de chapelain in FEW, II, 1, 286a, cappella: "prêtre chargé de dire la messe dans une chapelle particulière ", définition nuancée par l'indication que dans de nombreux textes en ancien français le chapelain apparaît comme le secrétaire du roi, sans que l'on puisse savoir s'il exerçait d'autres fonctions, spirituelles, celles-là. La polysémie de chapelain et de son étymon cappellanus est liée à celle de cappella, "petite chape ", qui désigne à l'origine (679) la relique du manteau de saint Martin, conservée dans un bâtiment construit pour l'abriter, puis par métonymie (710) le bâtiment lui-même et enfin, dès 783, une chapelle privée, se substituant dans ce sens à oratorium. Au VIII siècle, les cappellani veillent sur les reliques; à partir du X $\mathrm{X}^{\mathrm{e}}$ siècle, ils sont chargés de la rédaction de la correspondance royale. Mais dès la fin du VIII ${ }^{\mathrm{e}}$ siècle, cappellanus peut aussi désigner un "prêtre chargé de dire la messe dans une chapelle particulière ». Chapelain figure au vers 2157 (Livre II) du Roman du Mont Saint-Michel avec son sens originel de " gardien des reliques» : Li abes Mainarz, del mostier Qui premiers out cure et mestier, Par le conseil de son convent, Qui l'otreierent bonement, Funt de Durant lor chapelain, Honestes clerc et non vilain. : «L'abbé Mainard, qui le premier eut la responsabilité et la charge de l'abbaye, après consultation de son couvent, qui y consentit volontiers, fit de Durant leur chapelain : c'était un clerc digne d'estime, qui ne manquait pas de qualités. »

32. Son dérivé, clergie, désigne, au v. 10 du Roman, l'instruction, le savoir, et même plus précisément, dans le contexte, la connaissance du latin, dont disposent les hommes d'Église. C'est pour cels qui escient n'unt de clerzie, "ceux qui n'ont pas le savoir des clercs » que l'auteur transpose en français l'histoire du Mont.

33. Il n'est pas fait mention dans ces vers de sceaux que brise le destinataire de la lettre, comme dans la scène précédente (v. 1801-1802). L'auteur du Roman a probablement voulu éviter à son lecteur la répétition de détails identiques; on peut cependant conjecturer que les lettres du pape et des cardinaux étaient scellées, afin de valider l'authenticité du message confié aux envoyés du duc.

34. Cf. FEW, III, 398a, faluppa, afr. voloper «envelopper» et desvoloper, desvoleper, desveloper: «étendre ce qui était roulé sur soi-même; ôter l'enveloppe qui couvre quelque chose». Cf. également le verbe desploier: "étendre ce qui est plié, roulé», in FEW, IX, 70b, plicare.

35. Avranches, Bibl. mun., ms 210, fol. $23 v^{\circ}$. 
Bueve de Hanstone, poème épique du XIII ${ }^{\mathrm{e}}$ siècle ${ }^{36}$. Dans ce cas, devoleper désignerait l'acte de sortir la lettre de la boîte ou de l'enveloppe qui la contient et desploier celui de la déplier pour la lire. Mais aucune indication ne permet ici de déterminer la forme précise prise par le brief.

Le texte de la lettre se présente alors au chapelain dans sa totalité. Il commence par l'esgarder (v. 1829), comme le pape l'avait fait précédemment (v. 1802) : il examine attentivement le document ${ }^{37}$ pour en prendre globalement connaissance. Le verbe entendre (v. 1832) complète et explicite esgarder: en effet, cet examen, cette étude de la lettre, permet au chapelain d'en comprendre le texte latin (c'est un des sens du verbe entendre en ancien français) avant d'en restituer en français le contenu à son maître. Deux opérations successives mentionnées également dans une lettre presque contemporaine du Roman, adressée à Thomas Becket par Nicholas, prieur du MontSaint-Jacques à Rouen, à la Noël 1164. Rendant compte à l'archevêque de Cantorbéry de son ambassade auprès de l'impératrice Mathilde à propos des Constitutions de Clarendon, il note: precepit nobis eas latine legere et exponere gallice « elle nous ordonna de les lire en latin et de les expliquer en français ${ }^{38}$.

36. Dans Bueve de Hanstone, poème épique du XIII' ${ }^{e}$ siècle (éd. Albert STIMMING, Dresde, Gesellschaft für romanische Literatur, 1912 et 1914, II, 241-276, p. 11-13), c'est un clerc qui est chargé de rédiger la lettre, et un autre clerc de la lire: Un clerc apele, qui ot non Salemon [...] «Fai moi un brief si le seele en plon, De moie part le porteras Doon [...] » Lors fist un brief a sa division; Quant il l'ot fait, si le seele en plon, En une boiste a mis le quareignon [...] (II, v. 241, p. 11, 252-253 et 262-264, p. 12); Doon trova sous le pin au perron, Tout entour lui estoient si baron. Cil le salue qui n'estoit pas bricon, Le briel li baille atout le quareignon Ou ert escrite la mortel traïson, Et Do le livre a un sien clerc Milon; Cil freint la cire et desconfit le plon, La letre liut, bien vit en la leçon, Les mos connut et la devision [...] (II, v. 269-277, p. 13): "Elle appela un clerc du nom de Salomon [...] : Fais-moi une lettre et scelle-la avec du plomb. Tu la porteras de ma part à Doon ». La même scène figure dans une autre version de l'œuvre (III, v. 165-200, p. 9-11). Cf. également FEW, II, 2, 1439a, quaternio ("papier plié en quatre») : afr. quaregnon "parchemin plié en quatre; document, lettre» (tous deux XII ${ }^{\mathrm{e}}$-XIII ${ }^{\mathrm{e}} \mathrm{s}$.). Le Dictionnaire de l'ancienne langue française et de tous ses dialectes, du IX $X^{e}$ au XV $V^{e}$ siècle, de Frédéric GODEFroY, Paris, Champion, 1880 (Réédition Kraus Reprints, Neudeln/Liechtenstein, 1969), I, 783a, précise les sens du substantif: «carré de parchemin ou parchemin plié en carré, pli renfermant une lettre, la lettre elle-même». Cf., cités par GODEFROY, ibid., ces vers du Rouman d'Alixandre, (1180): Et quant il [le brief] est ploies et mis en quareignon Sel met on en.i. cofre [...], où le verbe ploier "plier» est associé à quareignon, qui a ici le sens de "pli renfermant une lettre» : «Et quand il est plié et mis sous pli (?), on le place dans un coffret [...].

37. Cf. FEW, XVII, 512b, *wardôn, afr. mfr. esgarder «faire attention à, remarquer, examiner». Dans le verbe dérivé comme dans le simple garder « veiller sur, prendre soin de » ou «faire attention à » on trouve le sens de «être attentif» de la base germanique war (présente aussi dans garir "protéger, défendre » et garnir « avertir, munir, fortifier»). On notera aussi la locution médiévale faire esgart: « examiner» et le substantif esgardee: «action d'examiner, de passer en revue». Le verbe esgarder figure avec le même sens dans un contexte identique au vers 2385 , à propos d'une donation de Richard II au Mont Saint-Michel, dont Guillaume de Saint-Pair s'apprête à donner la teneur, d'après le texte latin de la charte qu'il a consulté dans le Cartulaire $\left(\mathrm{ms} 210\right.$, fol. $\left.20 \mathrm{r}^{\circ}\right)$ : La chartre en ont encor li moine Qui toz ses dons lor testemoine. Ge la lui ja et esgardei. Or vos disrai que i trovai. (v. 2383-2386): «Les moines en conservent encore la charte qui leur garantit tous ses dons. Je l'ai lue et examinée et vais vous dire ce que j'y ai trouvé. »

38. The Correspondence of Thomas Becket, Archbishop of Canterbury, 1162-1170, éd. et trad. Anne J. DugGan, Oxford, Clarendon Press, 2000, Volume I, lettres 1-175, lettre 41, p. 166. Cf. CHIBNALL, 
La lecture-traduction débute par la formule officielle de début de lettre, les saluz (v. 1831) ${ }^{39}$, terme qui résume schématiquement le protocole; puis le chapelain traduit le texte (Puis a tot leit le brief avant, v. 1833), d'un bout à l'autre (avant précise que la lecture se fait du début vers la fin), jusqu'au valei qui représente l'eschatocole ${ }^{40}$. Dans cette lecture en trois parties, l'auteur précise qu'il ne falli ne tant ne quant (v. 1834): il ne se trompa absolument pas, c'est-à-dire ne commit aucune erreur de traduction.

Guillaume de Saint-Pair est à même d'en juger, puisque dans le texte le clerc et lui-même ne font qu'un : la lecture-traduction de la charte du pape Jean XIII, que le chapelain est censé faire à son maître, est présentée par ses soins au lecteur aux vers 1835-1847. Ce passage, détaché nettement du récit au passé simple qui le précède et qui le suit par l'emploi de l'imparfait descriptif puis du présent, résume le contenu de la lettre et permet au moine, en actualisant les excommunications et malédictions du pape, d'indiquer qu'elles continuent de s'appliquer aux contrevenants de son époque.

Peu importe que cette charte soit un faux, fabriqué par les moines du XI' siècle pour appuyer leur volonté d'indépendance face au pouvoir de l'évêque d'Avranches et à celui du duc de Normandie ${ }^{41}$. Guillaume de Saint-Pair, qui disposait de ce texte dans le Cartulaire ${ }^{42}$, entendait bien rendre compte d'un document si favorable à l'abbaye et aux moines, qui les autorisait à choisir leur abbé au sein de leur propre communauté, conformément au chapitre 64 de la Règle de saint Benoît. Il le fait en deux fois : ici, il résume brièvement le début de la lettre du pape; plus loin, aux vers 2279-2322, il fournit les détails du privilège, qui enregistre l'avantage accordé par Jean XIII aux moines du Mont; il précise alors qu'il revient à un sujet abordé précédemment ${ }^{43}$.

Marjorie, The Empress Matilda, Queen Consort, Queen Mother and Lady of the English, Oxford, B. Blackwell, 1991, p. 169-172. Je remercie vivement Elisabeth Van Houts d'avoir attiré mon attention sur ce document qui présente, à propos de la lecture d'une lettre, des traits communs avec la scène du Roman.

39. Cf. FEW, XI, 126a, salus « formule exclamative de souhait, de civilité, employée spécialement dans le préambule des lois et ordonnances, dans les lettres patentes des rois, dans les bulles des papes, etc.».

40. Termes employés par Jean FAVIER, Dictionnaire de la France médiévale, Paris, Fayard, 1993, p. 252253 , article «charte »; « [...] protocole: « invocation, titulature, c'est-à-dire nom et titre de l'auteur, adresse générale ou particulière, notification, exposé ou historique de l'affaire, dispositif»; eschatocole: «formules pieuses, annonce des signes de validation, date de lieu et de temps»; le texte lui-même se présente généralement avec "préambule général, notification, exposé ou historique de l’affaire, dispositif - c'est ce qui donne son sens à l'acte : don, vente, concession, etc. - et clauses juridiques finales $[\ldots] »$.

41. Cf. KeATS- ROHAN, «Une charte de l'abbé Mayeul... ».

42. Le texte figure dans l'Introductio monachorum sous le titre suivant: Sacrum domni Papce Johannis

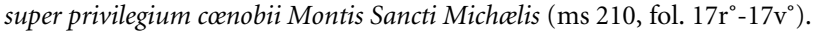

43. Cf. Livre II, v. 2279-2281: Del priviliege a l'apostoile Vos revoil or faire memoire; Johan out non, produen esteit: «Je veux à présent vous rappeler le privilège accordé par le pape; il se nommait Jean et c'était un homme de bien». Les vers 2282-2322 résument le texte de la lettre. 
Le moine du Mont offre ainsi à son lecteur un témoignage sur ce point précis de la lecture d'une lettre au XII siècle. Il le séduit par une de ces digressions, en apparence anecdotiques, dont il est coutumier et qui contribuent à l'intérêt et même au charme de son ouvrage. Tout cela sans jamais perdre de vue la réalisation d'un projet qu'il n'explicite pas, mais qu'il poursuit habilement et opiniâtrement et qui se dévoile tout particulièrement dans ce livre II du Roman du Mont Saint-Michel: défendre auprès de ceux qui le liront les droits de «sa » maison et de «ses » compagnons ${ }^{44}$.

44. Roman du Mont Saint-Michel, Livre II, v. 2652 : la meison (= «l'abbaye») et Livre III, v. 4000 : [les] norriz de la meson Qui esteient mi compaignon: «ceux qui avaient été éduqués au sein de la maison et qui étaient mes compagnons". 
